\title{
STUDY OF THE EFFECT OF VELOCITY ON END-TO- END DELAY FOR V2V COMMUNICATION IN ITS
}

\author{
Prof. Bhagat Sunilkumar Madhusudan ${ }^{1 \#}$ Prof (Dr.) Wadhai V.M ${ }^{2}$ \\ ${ }^{1}$ Research Scholar (Amravati University), Dept. of Information Technology, MAE \\ Pune,India \\ ${ }^{2}$ Principal MITCOE, Pune, India \\ ${ }^{\#}$ Corresponding Author : bhagatsunilmegmail.com
}

\begin{abstract}
This paper focuses on vehicle to vehicle $(\mathrm{V} 2 \mathrm{~V})$ communications in VANET. With the development of vehicles and mobile Ad Hoc network technology, the Vehicle Ad hoc Network (VANET) has become an emerging field of study. VANET is a special case of MANET, and will play an important role in city road traffic control. It is a challenging problem for searching and maintaining an effective route for transporting data information. Vehicular Ad Hoc network (VANET), is a hot topic applying mobile Ad Hoc network (MANET) to ITS in recent years. In the era of wireless computing, VANET communications are gaining a lot of attention. In this paper we provide a simulation and study of the effect of velocity on end-to-end delay for $v 2 v$ communication in intelligent transportation Systems
\end{abstract}

\section{KEYWORDS}

Vehicular networks, V2R communication, VANET, WiMAX, wireless networks.

\section{INTRODUCTION}

Inter Vehicle communication has been the new challenge in mobile networking. Vehicular communication can help in accumulating information, which shall be used to enhance traffic safety applications by monitoring weather conditions, reducing causalities by introducing intersection safety, break warnings and collision assistance. Most of the applications related to vehicle-to-vehicle communication need good connectivity to other vehicles and to the communicating infrastructure. This exacts reliable, delay and disconnection tolerant networks. Owing to the fact that mobile nodes are dynamic in nature, they also need to adapt to the newly entering nodes and thus impose many challenges to such networks. End-to-end delay refers to the time taken for a packet to be transmitted across a network from source to destination. Low packet delivery ratio is a big obstacle for inter vehicle communication Thus if support from existing infrastructure is taken and a new routing mechanism is devised so as to intermix between mobile nodes and existing Access Points then the future dream of many vehicle to vehicle communication dependent applications will come true. It is difficult, in terms of infrastructure cost, to cover roads with a large number of RSUs so that every vehicle on road can always be connected to at least one nearby RSU. Instead, vehicle-to-vehicle communications should be used in a multihop fashion in order to allow vehicles to connect to the out-of-transmission range RSUs, with a reasonable number of RSUs covering the road as shown in Figure 1. [1, 2, 3] 


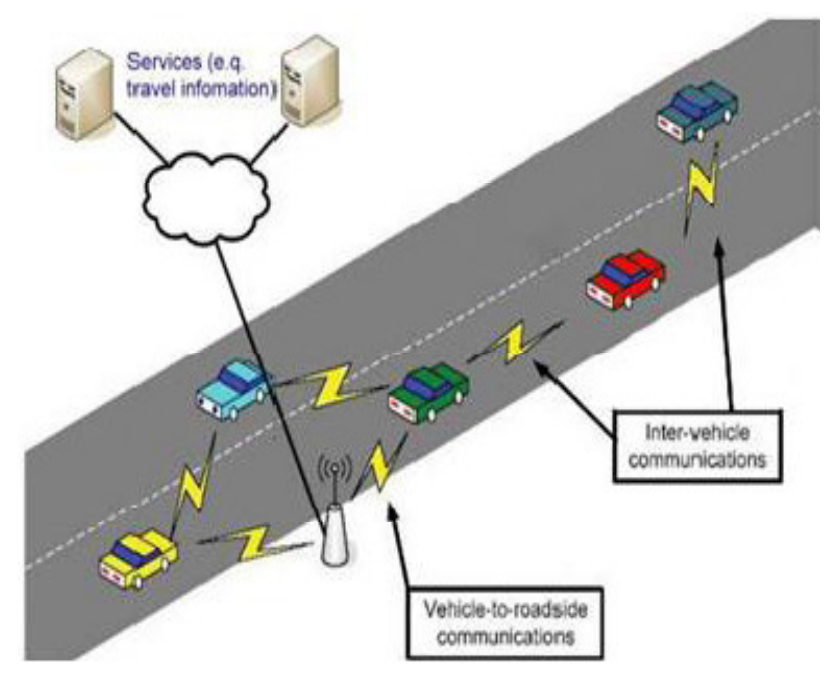

Figure 1. Architecture

\section{RELATED WORK}

Because of the high mobility of vehicles, it is a very challenging problem to find and maintain routing information in VANET, which is also one of the hottest research points. There are many research results have been put forward which can be divided into five categories: traditional ad hoc routing such as Dynamic Source Routing (DSR)and Ad-hoc On demand Distance Vector (AODV), but many researches show that most of them have poor performance when used in the vehicle network; Position based routing: due to restrictions on vehicles' movement on roads and the general two directions, using the information from the Global Position System (GPS) in routing strategy of the vehicles is feasible and effective. There are many researches in this area such as Greedy Perimeter Stateless Routing (GPSR), Greedy Perimeter Coordinator Routing (GPCR) and so on; Cluster based routing: due to vehicles move restricts on the roads and generally two directions, we can divide the vehicles into groups to improve scalability, each group have a header which is responsible for the coordination of groups and the nodes in his group. Clustering for Open IVC Networks (COIN) and Location Routing Algorithm with ClusterBased Flooding (LORA_CBF) are belonging to these protocols; Broadcast based routing: broadcasting in the distribution used frequently to notify a variety of common information and in the unicast routing protocols such as route discovery also use broadcast to find a valid path. Urban Multi-Hop Broadcast protocol (UMB) is one of the protocols that use broadcast to distribute packets. All broadcast based protocols need to consider the broadcast storm problem: Geocast routing such as Inter-Vehicles Geocast protocol (IVG) and Robust Vehicular Routing (ROVER), like position based multicast protocol, the basic idea of which are distributing message from the source node to some nodes in a special geographical region[1,4,5,6,7].

\section{CONCEPT}

Our aim is to provide a solution specific to existing VANET scenario to study effect of velocity on end to end delay between V2V (Vehicle to vehicle) communication. Implementation of V2I 
(Vehicle to Infrastructure) are also tried in simulation in wireless domain this in turn will enable building a VANET system with the following objectives:[9,10,11,12,13,14]

- To develop a system to study the impact of velocity on moving vehicles in terms of loss of connections and packets.

- How the congestion affect the delivery of packets in terms of dense networks.

- To study how to overcome frequent loss of connection.

In our work we are using a network simulator, QualNet 5.1 as shown in Figure 2. QualNet provides the simulation environment in order to simulate the entire wireless system when applied to vehicular communication in order to make vehicles intelligent. As our aim is to reduce End-toEnd delay we are concentrating on 'velocity' parameter. As QualNet does not provide a way to differ the velocity as per our requirement, We have implemented our own module to do so.

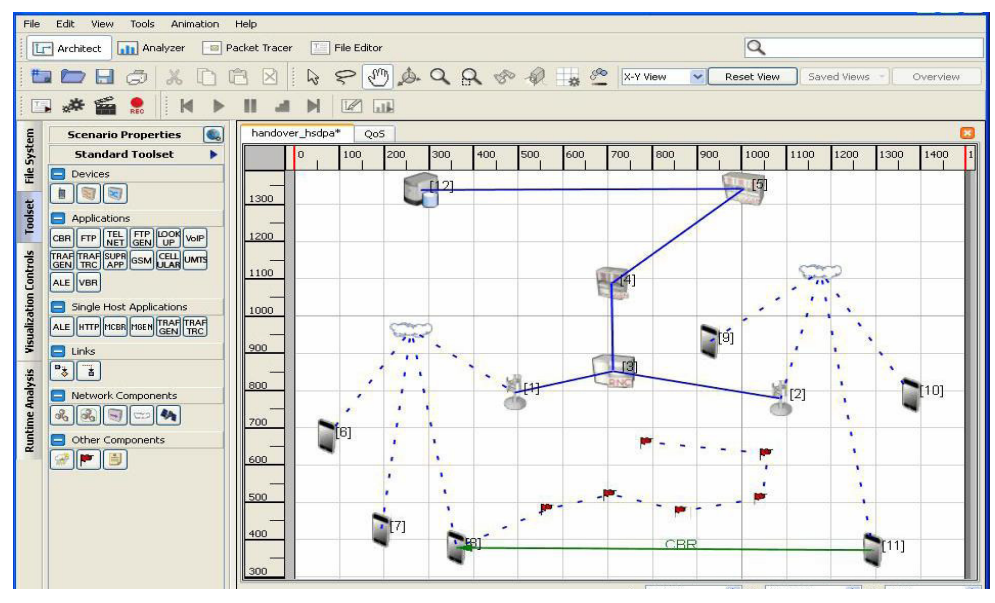

Figure 2. QualNet 5.1 Design mode

\section{Project Implementation}

The simulated work which we have developed for showcasing V2V communications in ITS has been done in the following sequence. We started our work in with deployment of VANET

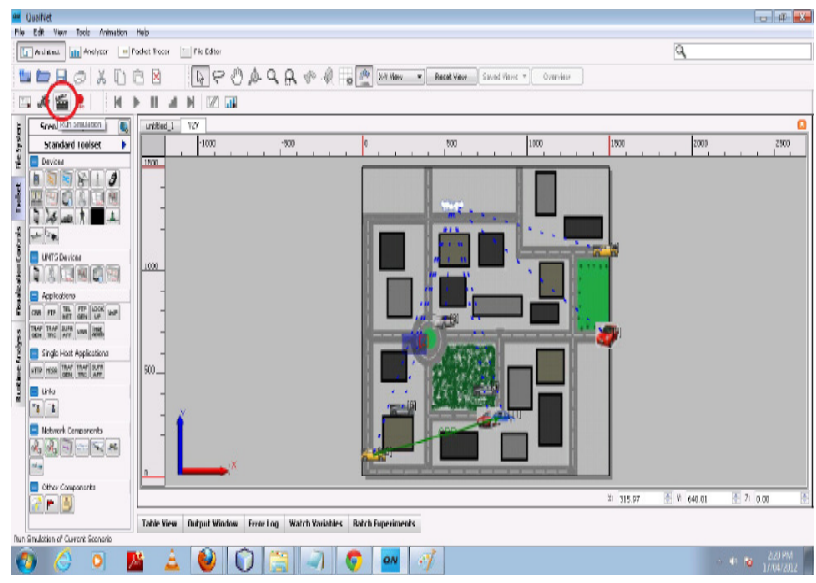

Figure 3. VANET in City environment 
Our actual aim was to show effect of velocity on end to end delay in V2V communication in ITS. As Qualnet does not provide functinoality so we have implimented as shown in Figure 4

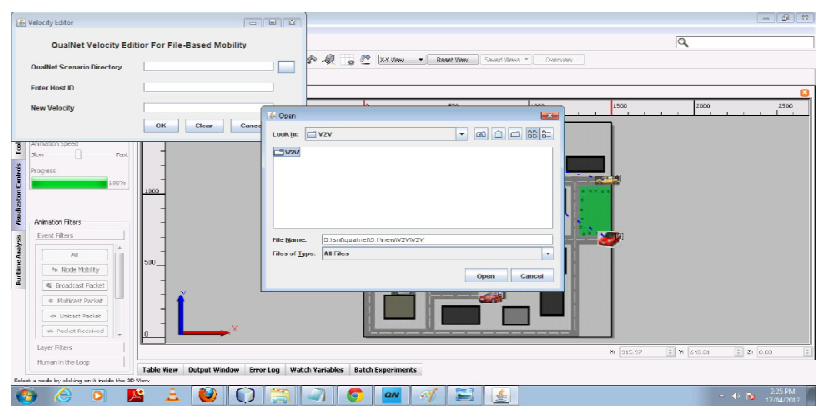

Figure 4. Velocity Editor

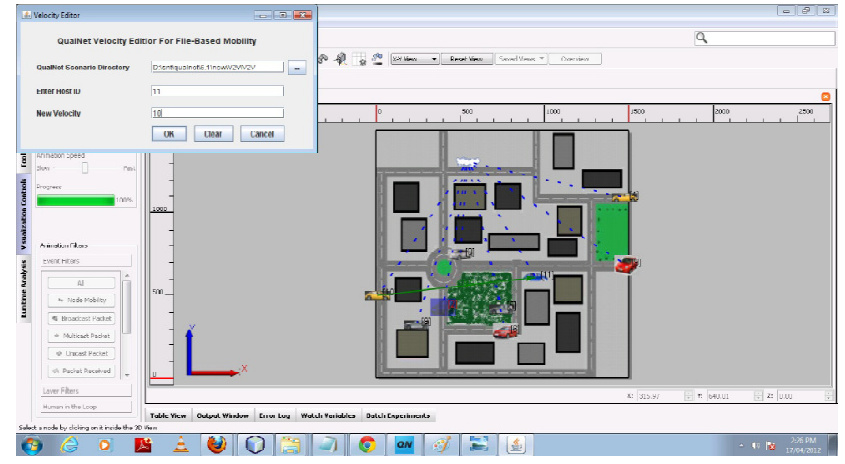

Figure 5. Setting Velocity through QualNet Velocity Editor

\section{RESULTS}

The simulation is run against different Velocity We go on changing the routing protocol to be used in our VANET scenario and for each protocol we simulate with a set of three different velocities to have a comparative study of end-to-end delay, throughput and total number of packets received To have a fair comparison, we keep the same number of packets to be sent in every simulation run for different routing protocol and different velocity. The entire simulation is shown in the following screenshots and results for the same which were analyzed are also shown.

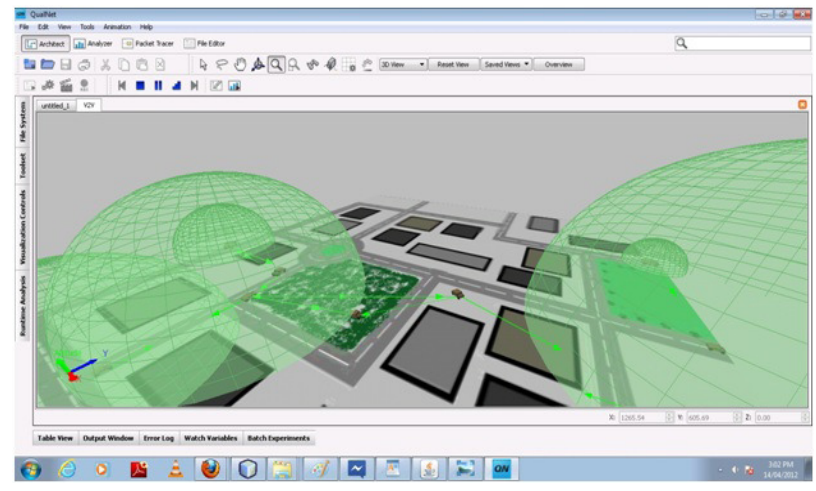

Figure 6. Initial arrangement of the vehicles 
To have a fair comparison, we keep the same number of packets to be sent in every simulation run for different routing protocol and different velocity.

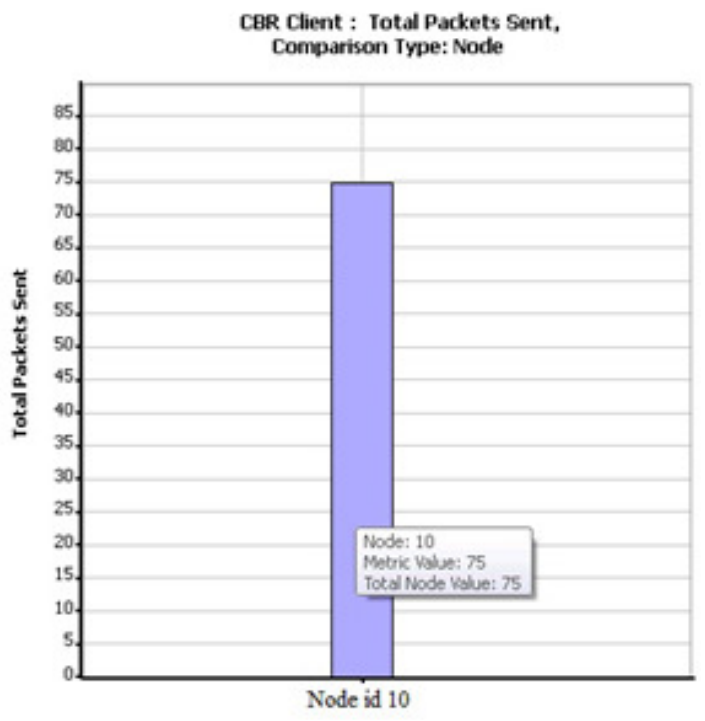

Figure 7. Packets sent by Node id 10

Here is a comparison of total number of packets received for three routing protocols viz. AODV, Bellman Ford and DSR where the source and destination vehicles move with a speed of 6 meter $/ \mathrm{sec}(21 \mathrm{~km} / \mathrm{hr})$.
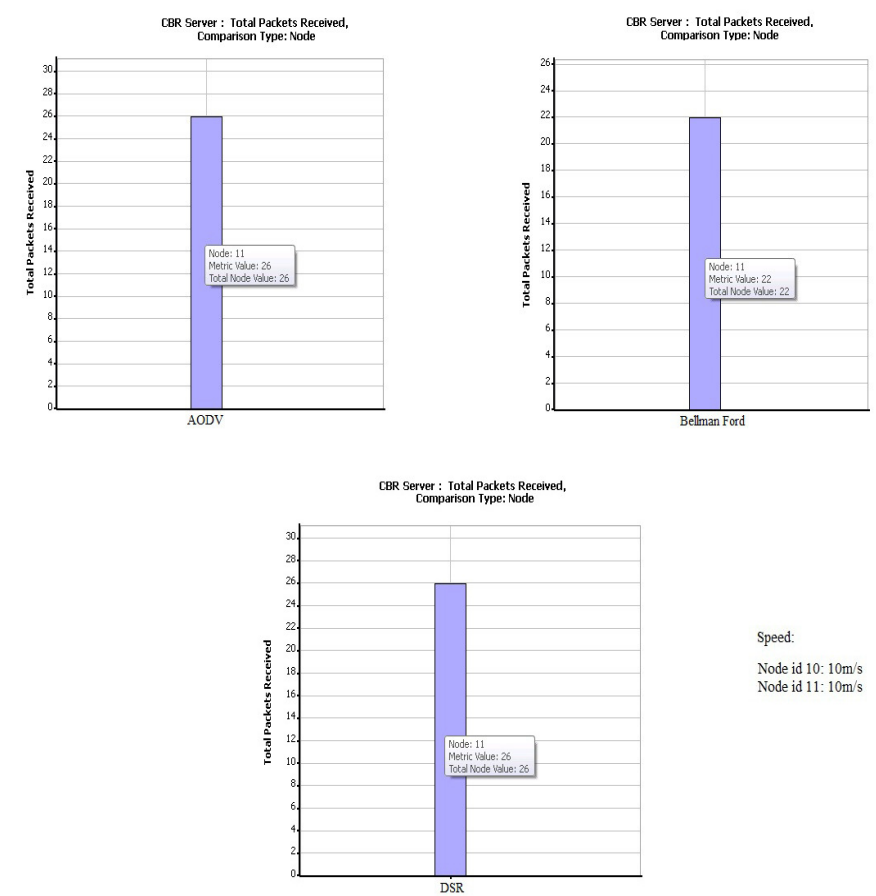

Figure 8. Packets Received for velocity $=06 \mathrm{~m} / \mathrm{s}$ 
International Journal of Next-Generation Networks (IJNGN) Vol.4, No.4, December 2012

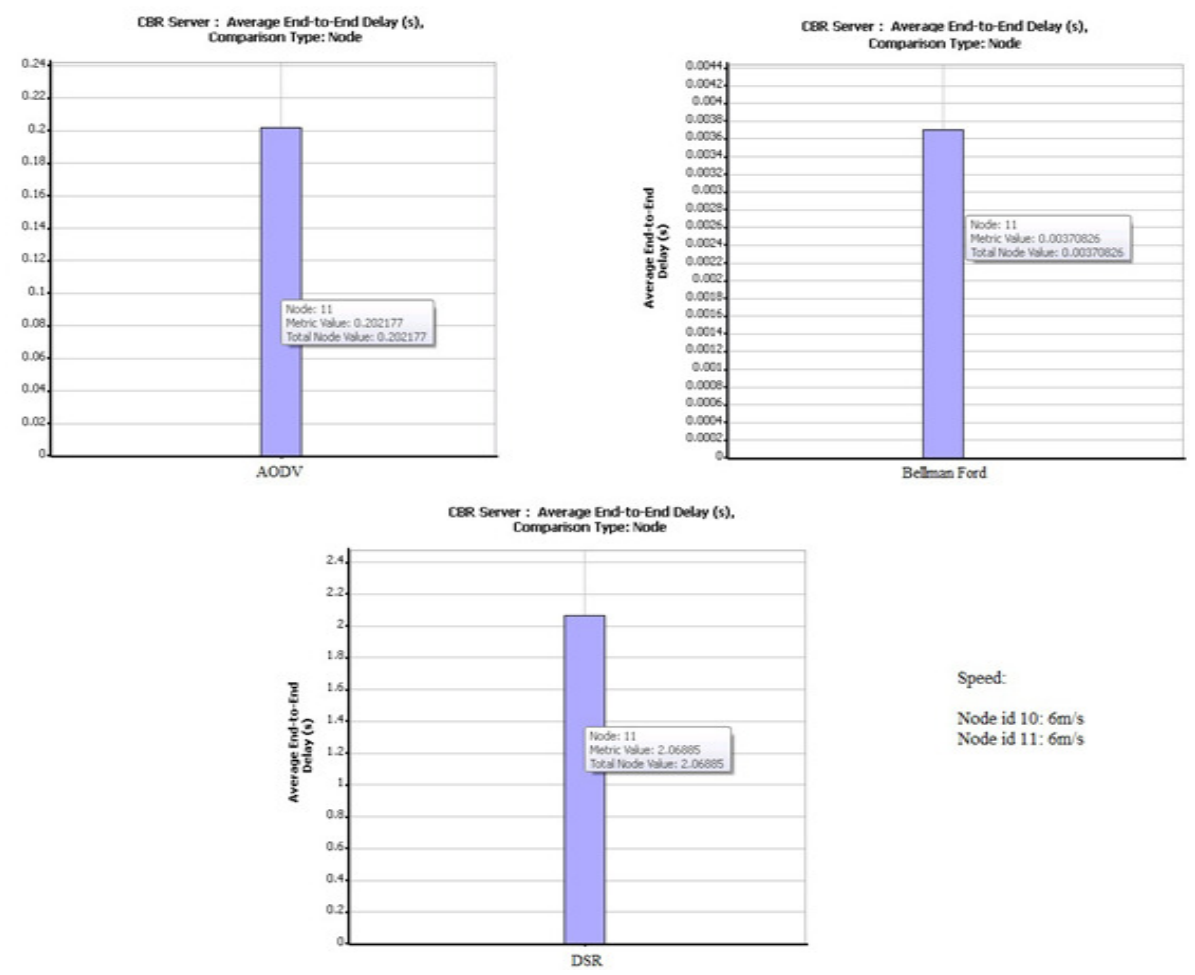

Figure 9. End-to-End Delay for velocity $=6 \mathrm{~m} / \mathrm{s}$

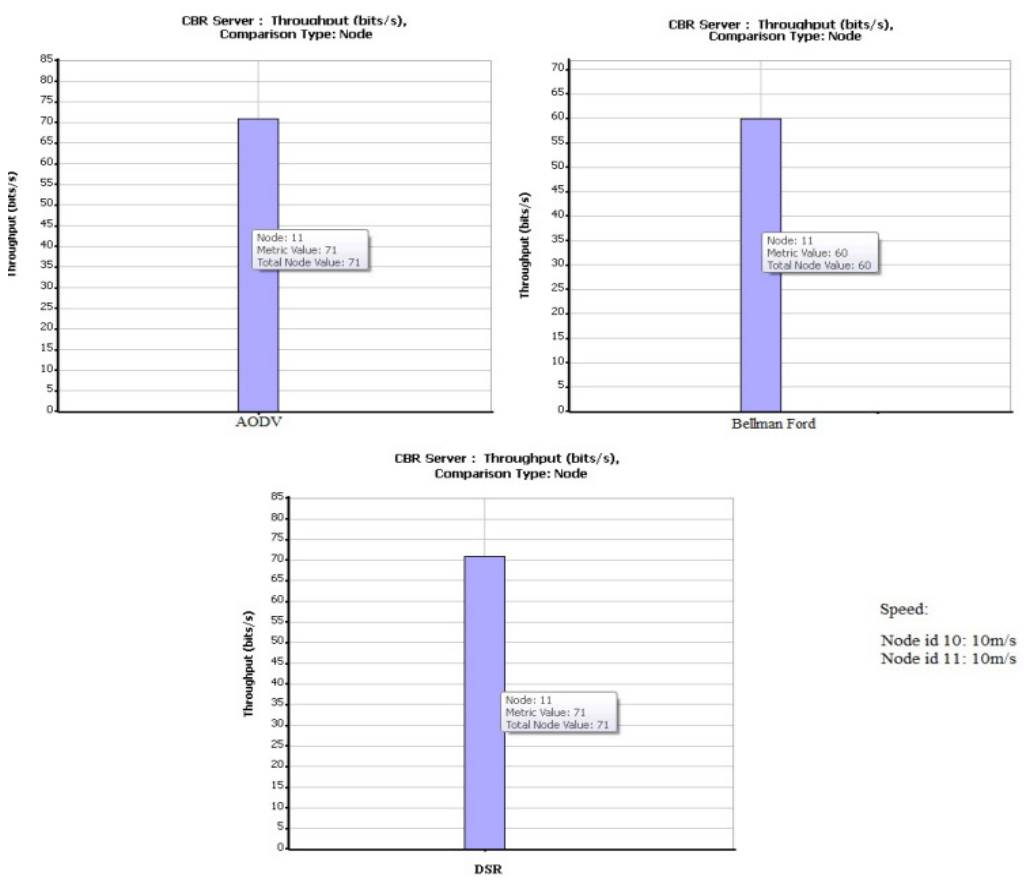

Figure 10. Throughput for velocity $=6 \mathrm{~m} / \mathrm{s}$ 


\section{Conclusions}

At the end of this work we conclude that we had studied various VANET routing protocols for V2V communication in urban scenario implemented using QualNet VANET scenario and it is found that performance of the Bellman ford routing protocol is better than other protocol for parameter end to end delay for all sets of velocity in urban scenario i.e. Bellman ford gives lowest end-to- end delay for all sets of velocity in urban scenario.

\section{ACKNOWLEDGEMENTS}

We take this opportunity to thank Dr. V. M. Thakare, Head Research Center, Amravati University for their valuable guidance and for providing all the necessary facilities.

\section{REFERENCES}

[1] James Bernsen, D. Manivannan "Unicast routing protocols for vehicular ad hoc networks: A critical comparison and classification" Journal on Pervasive and Mobile Computing in 2009 pp 1-18.

[2] Ming-Fong Jhang, "On Cooperative and Opportunistic Channel Access for Vehicle to Roadside (V2R)”, 2008 IEEE, "GLOBECOM" 2008 proceedings

[3] Qing Yang, Alvin Lim, Shuang Li, Jian Fang, Prathima Agrawal "ACAR: Adaptive Connectivity Aware Routing for Vehicular Ad Hoc Networks in City Scenarios" Mobile Networks and Applications Volume 15 Issue 1, February 2010, pp 36-60

[4] Yuyi Luo Wei Zhang Yangqing Hu "A New Cluster Based Routing Protocol for VANET", IEEE conference on Networks Security, Wireless Communications and Trusted Computing 2010 pp 176181.

[5] Yanlin Peng, J. Morris Chang “A Novel Mobility Management Scheme for Integration of Vehicular Ad Hoc Networks and Fixed IP Networks", ACM Journal on Mobile Networks and Applications, Volume 15 Issue 1, February 2010, pp 112-125

[6] Ikbal Chammakhi Msadaa, Pasquale Cataldi and Fethi Filali "A Comparative Study between 802.11p and Mobile WiMAX-based V2I Communication Networks", IEEE International Conference on Next Generation Mobile Applications, Services and Technologies 2010

[7] Fan Li and Yu Wang, University of North Carolina at Charlotte "Routing in Vehicular Ad Hoc Networks: A Survey” IEEE Vehicular Technology Magazine, June 2007, pp 12-23

[8] Yasser L.Morgan "Managing DSRC and WAVE Standards Operations in a V2V Scenario" International Journal of Vehicular Technology Volume 2010, pp 1-18

[9] Kevin C. Lee, Uichin Lee, Mario Gerla“Survey of Routing Protocols in Vehicular Ad Hoc Networks", IEEE conference on VANET 2008

[10] C. A. T. H. Tee, A. Lee "A Novel Routing Protocol - Junction Based Adaptive Reactive Routing (JARR) for VANET In City Environments”, IEEE Conference on European Wireless 2010, pp 1-6

[11] Li-Der Chou, Jyun-Yan Yang, Ying-Cheng Hsieh and Chi-Feng Tung "Intersection-Based Routing Protocol for VANET" IEEE conference on ICUFN 2010, pp 268-273

[12] Wenjie Shu, Ping Wang, Aihuang Guo, Xinhong Wang, Fuqiang Liu "Enhanced GPSR using Neighbor-Awareness position Update and Beacon-assist Geographic Forwarding in vehicular ad hoc networks" IEEE conference on Intelligent Vehicles Symposium, 2009 1143-1147

[13] Omid Abedi, Mahmood Fathy, Jamshid Taghiloo "Enhancing AODV Routing Protocol Using Mobility Parameters in VANET" IEEE International Conference on computer system and application 2008 pp 229-236

[14] Moez Jerbi,Sidi-Mohammed Senouci, Tinku Rasheed, and Yacine Ghamri-Doudane"Towards Efficient Geographic Routing inUrban Vehicular Networks" IEEE Transactions On Vehicular Technology, Vol. 58, No. 9, November 2009, pp 5048-5060

[15] Harsch, C., Festag, A., Papadimitratos, P. (2007). Secure position-based routing for VANETs. In Proceedings of IEEE 66th vehicular technology conference (VTC-2007), Fall 2007 (pp. 2630), September 2007.' 


\section{AUTHORS}

Mr. Bhagat Sunilkumar M. is Ph.D. Scholar of Amaravati University. He has received M.E. degree in Computer Engineering from Shivaji University, Kolhapur and B.E. degree in Computer Engineering from Amaravati University in 1990. He has experience of 22 years in academics and research and has been working on VANET routing protocol. His research interest includes Wireless Networking, Internet Bandwidth. Management. He acts as reviewer for international conference and serves as program committee member for ROBOCON. Currently he is working as a Professor

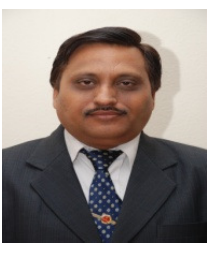
and Head, Department of Information Technology, MIT Pune's Maharashtra Academy of Engineering, Pune.

Dr. Vijay M. Wadhai received his B.E. from Nagpur University in 1986, M.E. from Gulbarga University in 1995 and Ph.D. degree from Amravati University in 2007. He has experience of 26 years which includes both academic (19 years) and research (7 years). He has been working as the Principal of MAEER's MITCOE, Pune since 2010. $\mathrm{He}$ is currently guiding various numbers of students for their $\mathrm{PhD}$ work in both Computers and Electronics \& Telecommunication area. His research interest includes Deductive Databases, Knowledge Discovery and Data Mining, Data Security, Natural

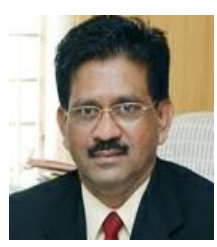

Language processing, Cognitive Radio and Wireless Communication, Spectrum Management, Wireless Sensor Network, ASIC Design - VLSI, Advance Network Design.. He has filed numbers of patents in his research areas and also published quality International journal papers. 